\title{
Magnetic nanoparticle targeted hyperthermia of cutaneous Staphylococcus aureus infection
}

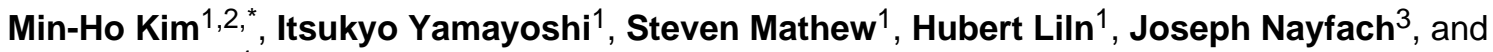 \\ Scott I. Simon ${ }^{1}$ \\ ${ }^{1}$ Department of Biomedical Engineering, University of California at Davis, Davis, CA95616 USA \\ ${ }^{2}$ College of Applied Engineering, Sustainability, and Technology, Kent State University, Kent, $\mathrm{OH}$ \\ 44242 USA
}

${ }^{3}$ Qteris, Inc., San Rafael, CA 94903 USA

\begin{abstract}
The incidence of wound infections that do not adequately respond to standard-of-care antimicrobial treatment has been increasing. To address this challenge, a novel antimicrobial magnetic thermotherapy platform has been developed in which a high-amplitude, high-frequency, alternating magnetic field (AMF) is used to rapidly heat magnetic nanoparticles that are bound to Staphylococcus aureus ( $S$. aureus). The antimicrobial efficacy of this platform was evaluated in the treatment of both an in vitro culture model of $S$. aureus biofilm and a mouse model of cutaneous $S$. aureus infection. We demonstrated that an antibody-targeted magnetic nanoparticle bound to $S$. aureus was effective at thermally inactivating $S$. aureus and achieving accelerated wound healing without causing tissue injury.
\end{abstract}

\section{Keywords}

antimicrobial thermotherapy; alternating magnetic field; $S$. aureus biofilm; magnetic nanoparticle

\section{Introduction}

Staphylococcus aureus ( $S$. aureus) has emerged as a leading cause of colonization and infection in various soft-tissue wounds including venous leg ulcers, pressure ulcers, and diabetic foot ulcers, resulting in the development of chronic, non-healing wounds ${ }^{1-4}$. Critical challenges in treatment of $S$. aureus infections include antibiotic resistance, and its multiple, redundant mechanisms of immune evasion such that $S$. aureus is able to escape many elements of innate or adaptive immune attack ${ }^{5-7}$. Biofilm formation is an important aspect of $S$. aureus immune evasion as is evidenced by the correlation between biofilm formation and the onset of wound chronicity 8 , .

Current approaches for the treatment of skin and soft tissue infections with $S$. aureus include the application of topical or systemic antibiotic treatments (i.e. clindamycin, trimethoprimsulfamethoxazole, tetracyclines, $\beta$-lactams, vancomycin, telavancin, daptomycin, and linezolid, among others) along with wound debridement, drainage, and surgical intervention ${ }^{10}$. Despite the development of novel antimicrobial agents, the cost and

\footnotetext{
"Corresponding author: Scott I. Simon, Department of Biomedical Engineering, University of California at Davis, 451 E. Health Sciences Dr., Davis, CA 95616, Phone: 530-752-0299, Fax: 530-754-5739, sisimon@ucdavis.edu.

Conflict of Interests: Joseph Nayfech (Qteris, Inc) declares competing financial interest.
} 
complexity of treating these chronic wound infections remain a serious challenge. A number of factors contribute to this, including extended treatment duration (i.e. up to several months) with concomitant nephrotoxicity and hepatotoxicity, a persistent growth of antibiotic-resistant bacterial strains, and a limited efficacy of antibiotics against bacterial biofilms ${ }^{11,12}$. To help address these challenges, we propose an adjuvant to antimicrobial treatment that could facilitate efficient and rapid inactivation of bacterial cells and biofilms without further compromising fragile patients or exacerbating drug resistance. We envision this treatment would facilitate reduced treatment durations and improve outcomes by acting synergistically to enhance the efficacy of existing antimicrobial agents.

This adjuvant treatment, termed antimicrobial magnetic nanoparticle (MNP) thermotherapy, is an evolution of targeted radio frequency (RF) hyperthermia ${ }^{13}$, which, through recent advancements in magnetic nanoparticle design, has proven successful in the treatment of various cancers including glioblastoma, prostate carcinoma, and breast carcinoma ${ }^{14-16}$. Targeted RF hyperthermia utilizes magnetic nanoparticles, which absorb electromagnetic radiation and efficiently transmit energy in the form of highly localized heat (i.e. nanometer range $)^{17}$. Motivated by aspects of this targeted ablation technology, we have applied it to thermally inactivate $S$. aureus within a cutaneous abscess in a mouse model of wounding.

Several other thermotherapy-based antimicrobial treatments have demonstrated modest antimicrobial efficacy using an application of infrared and light pulsing ${ }^{18,19}$, direct-current electrical stimulation ${ }^{20,21}$, ultrasound ${ }^{22,23}$, and alternating electric fields ${ }^{24}$. However, such approaches have been non-specific, frequently necessitating undesirable increases in ambient tissue temperature to generate bacterial cell destruction. Further, although antimicrobial photo-thermal treatments have demonstrated some efficacy ${ }^{25,26}$, these methods were similarly limited due to nonspecific tissue heating and due to the restricted tissue penetration depth of pulsed laser light.

The opportunity to thermally inactivate $S$. aureus depends upon its temperature sensitivity and its ability for thermo-tolerance ${ }^{27-29}$. It has been shown that heating $S$. aureus in tryptic soy broth (TSB) solution at $60^{\circ} \mathrm{C}$ for 20 min resulted in a $\sim 3 \log$ reduction, while heating $S$. aureus at $70^{\circ} \mathrm{C}$ for 2 min resulted in a $\sim 5 \log$ reduction ${ }^{29}$. Thomas et al. ${ }^{30}$ recently demonstrated in an in vitro culture model of $S$. aureus that magnetic fluid hyperthermia could be used to enable bacterial destruction.

The ability to safely heat bacterial cells and prevent tissue damage depends upon targeted heating of pathogen cells, facilitated by proper customization of treatment parameters. Although magnetic nanoparticle hyperthermia can generate a rapid temperature increase at the surface of a single $\mathrm{MNP}^{31}$, a theoretical study also showed that overdosing presents the potential for disseminated tissue heating due to simultaneous heat dissipation from a large number of MNPs dispersed in a macroscopic region of tissue ${ }^{32}$. Therefore, proper dosing and customization of magnetic field exposure parameters are necessary to facilitate a targeted temperature rise, and prevent undue tissue damage to the surrounding tissue.

In this study, we developed and validated a MNP thermotherapy platform that could specifically target and kill bacteria in an in vitro culture model of $S$. aureus biofilm and in a mouse model of cutaneous $S$ aureus infection. First, a custom electro-magnetic (EM) generator was designed in which a short-duration exposure of a high-frequency, highamplitude alternating magnetic field (AMF) could deliver high specific loss power (SLP) to MNPs. Second, anti-protein-A antibody was attached to magnetite $\left(\mathrm{Fe}_{3} \mathrm{O}_{4}\right)$ nanoparticles to facilitate specific binding of the nanoparticle conjugates to the surface coat of $S$. aureus and direct targeted heating for thermal inactivation. 


\section{Materials and Methods}

\section{SLP measurement}

SLP provides a measure of the energy dissipation by a MNP to the tissue and is defined as the thermal power dissipated by a material per unit mass during exposure to an AMF. The SLP value was calculated using $\mathrm{SLP}=\left(\mathrm{CV}_{\mathrm{S}} / m\right)^{*}(\mathrm{dT} / \mathrm{dt})^{13,33}$, where $\Delta \mathrm{T} / \Delta \mathrm{t}$ is the initial rate of the temperature rise $(\mathrm{T})$ during the heating interval $(\mathrm{t}), \mathrm{C}$ is the volumetric specific heat capacity of the sample solution $\left(4.186 \mathrm{~J} /\left(\mathrm{g}{ }^{\circ} \mathrm{C}\right)\right.$ for water), $\mathrm{V}_{\mathrm{s}}$ is the sample volume, and $m$ is the mass of magnetic material in the sample. The $200 \mu \mathrm{g}$ of MNP (100 nm nanomag-DSPIO, iron concentration of $0.48 \mathrm{mg}$ per $\mathrm{mg}$ particle) was added to the well with $1 \mathrm{~mL}$ water. The sample well was then inserted in a water-cooled magnetic coil and a fiber optic temperature probe (Neoptix) was placed in the solution for continuous monitoring of temperature during the AMF exposure. AMF was applied at a frequency of $2.1 \mathrm{MHz}$ and at a field strength of 18,31 , and $40 \mathrm{kA} / \mathrm{m}$. A time-dependent change in temperature in the solution was continuously monitored using the fiber optic temperature probe.

\section{AMF-mediated MNP heating to disrupt $S$. aureus biofilm in vitro}

Bioluminescent $S$. aureus (SH1000 strain; generously provided by Dr. Ambros Cheung at Dartmouth Medical School) was streaked onto Tryptic soy agar (Tryptic soy broth [TSB] + $1.5 \%$ Bacto Agar) and grown overnight at $37^{\circ} \mathrm{C}$ in a shaking incubator $(240 \mathrm{rpm})$ as previously published ${ }^{34,35}$. Colony-forming units (CFUs) were verified by plating dilutions of the inoculum onto TSB agar \pm chloramphenicol overnight. To develop biofilms, $100 \mu \mathrm{L}$ of stationary growth phase $S$. aureus culture was added to a well of a tissue culture-treated polystyrene 96 -well plate under aseptic conditions, as previously described ${ }^{36,37}$. Biofilms were developed (at $37^{\circ} \mathrm{C}$ ) for $24 \mathrm{hrs}$, and any non-attached cells were rinsed off with sterile PBS. Then, a defined amount of MNP was added to the well and an AMF of variable amplitude was applied. To study the antibody-linked MNP targeting of $S$. aureus biofilm, a defined amount of antibody-linked MNP $(200 \mu \mathrm{g})$ was added to the biofilm and incubated for $2 \mathrm{hrs}$ at room temperature. Any unbound, free antibody-linked MNPs were subsequently removed by repeated washes with sterile PBS, and a defined AMF (at a field strength of $31 \mathrm{kA} / \mathrm{m}$ for $3 \mathrm{~min}$ ) was applied. $S$. aureus inactivation was assessed based on the quenching of bioluminescence signal before and after an AMF treatment using IVIS 100 imaging system (Caliper Life Science, Hopkinton, MA). Analyses of images were performed using Live Image Pro 2.5 (Caliper Life Science, Hopkinton, MA).

\section{Flow cytometric assay of anti-protein A antibody binding to $\mathrm{S}$. aureus}

Biotinylated anti-protein A antibody (Abcam, MA) and isotype control (mouse IgG1, eBioscience, CA) were conjugated with streptavidin PE-Cy5 (eBioscience, CA). To assess the binding of anti-protein A antibody to $S$. aureus, $1 \times 10^{7} \mathrm{CFU}$ of $S$. aureus (in $100 \mu \mathrm{L}$ suspension) were stained with either PE-Cy5 conjugated anti-protein A antibody or PE-Cy5 conjugated IgG1 for $20 \mathrm{~min}$ on ice. Events were acquired using a FACScan flow cytometer (Becton Dickinson, NJ). Data was analyzed using FlowJo software (Treestar, Inc., OR).

\section{Conjugation of anti-protein A antibody with MNP}

For the attachment of antibody to MNP, we employed a method of biotin-streptavidin binding (Fig. 1). We used commercially available streptavidin-coated MNPs (nanomag-Dspio, micromod $\mathrm{GmbH}$, Germany) for attachment to either biotin conjugated anti-protein A antibody (Abcam, MA) or isotype control (mouse IgG1, eBioscience, CA). In a preliminary experiment, an optimal concentration of antibody for linking with MNPs was determined by quantifying the half maximal concentration of antibody bound, which was found to be $75 \mu \mathrm{g}$ antibody/mg MNP. This value was used for the entirety of the antibody-targeting 
experiments (data not shown). After incubation of the antibody with MNP for $1 \mathrm{hr}$ at room temperature, any unbound free antibody was washed out by repeated centrifugation three times and reconstituted with sterile PBS for further use for in vitro and in vivo experiments.

\section{In vitro binding efficiency of antibody-targeted MNPs to $S$. aureus biofilm}

The binding efficiency of anti-SA MNPs to $S$. aureus biofilm was quantified by determining the total quantity of MNPs remaining after removal of free, unbound MNPs by repeated washes. The total quantity of remaining MNPs bound to $S$. aureus biofilm was quantified using a spectrophotometer (O.D at $490 \mathrm{~nm}$ ) after bleaching out the biofilm. The measured O.D value was correlated with a titration curve of MNP amount versus O.D value.

\section{S. aureus viability assay using SYTO 9-propidium iodide (PI) staining}

To assess the viability of $S$. aureus following AMF treatment, a SYTO9-PI bacterial viability kit (Invitrogen, CA) was used. A $3 \mu \mathrm{L}$ volume of an equal proportion of SYTO9 and PI mixture was added to the AMF-treated $S$. aureus biofilm on 96 well plates and incubated for $15 \mathrm{~min}$ in the dark at room temperature. After gently disrupting the biofilm structure with multiple pipetting, $10 \mu \mathrm{L}$ of stained suspension was spotted onto a microscope slide, which was examined using a Nikon epi-fluorescence microscope at $40 \mathrm{X}$ magnification with oil-emersion. Live cells (SYTO9) were observed with a $488 \mathrm{~nm}$ excitation, $535 \mathrm{~nm}$ bandpass emission filter, and dead bacteria (PI) were seen with a $488 \mathrm{~nm}$ excitation, $580 \mathrm{~nm}$ emission filter. The images were analyzed using Image $\mathrm{J}$.

Mice

EGFP-lysozyme M (lys) knock in-mice (EGFP-lys-mice) ${ }^{38}$ (kind gift from Dr. Thomas Graf at the Center for Genomic Regulation in Barcelona, Spain) were backcrossed for 10 generations onto a C57BL/6 background (Jackson Lab, Bar Harbor, ME) in an animal facility at University of California at Davis. Female mice between 8 and 14 weeks of age were used in all the experiments. All animal experiments were approved by Institutional Animal Care and Use Committee (IACUC) of the University of California at Davis and performed following the guidelines of the Animal Welfare Act and the Health Research Extension Act.

\section{AMF-mediated MNP thermotherapy of in vivo mouse model of $S$. aureus wound infection}

The induction of skin wound and $S$. aureus infection was performed as described in our previous studies ${ }^{34,39}$. Briefly, a circular, full thickness wound excision ( $6 \mathrm{~mm}$ in diameter) was made on the dorsal skin of a mouse using a skin biopsy punch (Robbins instruments Inc., NJ), and that mouse skin wound was then inoculated with a sub-lethal dose $\left(1 \times 10^{7}\right.$ CFU) of a bioluminescent strain of $S$. aureus. On day 2 post wounding and infection, $200 \mu \mathrm{g}$ of antibody-conjugated MNPs dissolved in sterile PBS were injected into the wound bed (Fig. 1). After 2 hrs, the mouse was placed in a magnetic coil and an AMF was applied for 3 min at a field strength of $31 \mathrm{kA} / \mathrm{m}$ and at a frequency of $2.1 \mathrm{MHz}$. The $S$. aureus burden in the wound was quantified using an IVIS 100 imaging system (Caliper Life Science, Hopkinton, MA), in which luminescence provided an enumeration of metabolically active $S$. aureus. S. aureus burden was measured during the course of infection (up to day 5 post wounding and infection) including before and immediately after AMF treatment. We monitored the ambient temperature within the wound area and surrounding non-wound area by infrared thermal imaging using a FLIR SC8000 thermal camera (FLIR system Inc., MA) before and immediately after AMF application. The wound size was determined by taking a photographic image of the wound area before and after AMF exposure using IVIS 100 imaging system and quantifying the image using Image $\mathbf{J}$. 


\section{Statistical analysis}

Data analysis was performed using GraphPad Prism version 5.0 software (GraphPad Software, San Diego, CA). Statistical significance was determined by two-tailedunpaired $t$ tests. $P$ values of $<0.05$ were considered statistically significant. Data are expressed as mean \pm SEM.

\section{Results}

\section{A high-frequency, high field amplitude thermotherapy platform}

For precise control over power delivery to MNPs during thermal inactivation of bacteria, a high-frequency, high-amplitude EM field generator was designed and fabricated with variable power (0-8kW) and RF signal (1.7 2.1 MHz) adjustment (Fig. 2a). This generator consists of a power source coupled to electromagnetic coils ( $40 \mathrm{~mm}$ in inner diameter). For proper cooling, chilled water is circulated through the electrical interconnects and the inner surface of the field coils. For further cooling, the field coils are housed within an enclosure that is filled with chilled, low-viscosity mineral oil that recirculates over a cold head. The maximum magnetic field applicator surface temperature was measured at $37^{\circ} \mathrm{C}$. The field generator did not produce any detectable higher order spectral components, and the total reflected power was less than $0.3 \%$. Operating at a maximum frequency of $\sim 2.1 \mathrm{MHz}$, the measured field amplitude inside the coil was in the range of $0-50 \mathrm{kA} / \mathrm{m}$. We assessed the efficiency of the EM generator to excite the MNPs and deliver thermal energy by quantifying the SLP as a function of field amplitude using $100 \mathrm{~nm}$ iron oxide particles at an applied frequency of $2.1 \mathrm{MHz}$, as described in Methods. SLP increased linearly with field strength from $\sim 20-40 \mathrm{kA} / \mathrm{m}$ and achieved a value of $900 \mathrm{~W} / \mathrm{g}$ at a field strength of $31 \mathrm{kA} / \mathrm{m}$ (Fig. 2b). This SLP magnitude was 9-fold greater than previously reported for similar MNP and field amplitude characteristics ${ }^{33}$, demonstrating that our custom EM generator was able to efficiently transfer thermal energy to MNP preparations.

\section{Efficacy of MNP thermotherapy on S. aureus biofilm disruption in vitro}

We evaluated the ability for MNP heating to effectively disrupt biofilm using an in vitro culture assay (Fig. 3). A biofilm of bioluminescent $S$. aureus was formed in 96 well plates, and the extent of bacterial killing was quantified based on the level of bioluminescence quenching that directly correlate with number of live bacteria (CFUs), as demonstrated previously ${ }^{35,40-42}$. Increasing amounts of MNPs $(0-200 \mu \mathrm{g})$ were added to each well with $S$. aureus and exposed to a defined magnetic field amplitude and frequency for 3 min. This brief AMF exposure disrupted biofilms in proportion to the amount of MNP in each well and the applied magnetic field amplitude (Fig. 3a). A 3-log reduction in S. aureus bioluminescence (about $99.9 \%$ killing) was achieved at a dose of $150 \mu \mathrm{g}$ MNPs at $40 \mathrm{kA} / \mathrm{m}$ and $2 \log$ reduction (about 99\% killing) at $200 \mu \mathrm{g}$ MNPs at $31 \mathrm{kA} / \mathrm{m}$. However, $18 \mathrm{kA} / \mathrm{m}$ was insufficient to significantly disrupt biofilms even at the highest dose of MNP at the 3 min exposure duration. This result demonstrated that brief AMF excitation of MNPs was sufficient to effectively disrupt biofilm and inactivate bacteria in vitro when using proper MNP dosing and AMF field parameters.

\section{Antibody-targeting of $S$. aureus biofilm in vitro}

We targeted protein A surface antigen on $S$. aureus using antibody-linked MNPs and evaluated the efficiency of bacterial killing upon high-frequency AMF heating. $S$. aureus universally expresses protein A on its surface ${ }^{6,43}$, which motivated our selection of antiprotein A antibody (anti-SA mAb) to target the SH1000 strain of $S$. aureus. We validated that anti-SA mAb had, in fact, bound specifically to $S$. aureus by detecting a significant increase in anti-SA mAb binding to $S$. aureus compared to that of an isotype control ( $\operatorname{IgG})$ 
(Fig. 4a). To assemble the targeting construct, streptavidin coated MNPs were conjugated to biotinylated anti-SA antibody. We subsequently determined the binding efficiency of the MNP-antibody conjugate to $S$. aureus biofilms, by measuring the percentage of MNPs that remained in the well after removing unbound MNPs by repeated washing. The measured maximum binding efficiency of anti-SA linked MNP to biofilm was $~ 50 \%$ using $200 \mu \mathrm{g}$ of MNPs (Fig. 4b). This bacterial targeting efficiency did not significantly increase with additional MNP-antibody added to the well and was more than 1-fold higher than that of the nonspecific MNP-IgG targeting at $\sim 20 \%$ binding efficiency. Next, we compared the extent of $S$. aureus biofilm disruption following AMF application (at a field strength of $31 \mathrm{kA} / \mathrm{m}$ for $3 \mathrm{~min}$ ) using the anti-SA linked MNPs versus anti-IgG isotype control linked MNPs (Fig. 4c). The data demonstrated more than a 1-fold enhanced $S$. aureus inactivation for antiSA targeting compared with either IgG in the in-vitro biofilm assay or non-targeted MNPs ( $\sim 70 \%$ killing for anti-SA vs $\sim 30 \%$ for IgG vs $\sim 30 \%$ for non-targeted MNPs at $31 \mathrm{kA} / \mathrm{m}$ field strength, $\mathrm{p}<0.05$ ) (Fig $4 \mathrm{c}$ and Fig.3a). The enhanced antimicrobial activity of anti-SA targeted MNPs was confirmed with a live/dead viability assay based on fluorescent staining of SYTO9 and PI (Fig. 4d). We conclude that anti-SA targeting significantly enhances $S$. aureus thermal inactivation in the in-vitro biofilm assay.

\section{In vivo validation of antibody targeted thermal inactivation of $S$. aureus to resolve infection and facilitate wound healing}

We validated the efficacy of MNP thermotherapy in vivo by employing a mouse model of $S$. aureus cutaneous wound infection $\left(1 \times 10^{7} \mathrm{CFU}\right)$. AMF treatment was applied when the $S$. aureus burden in the wound reached its maximum, which was demonstrated in our previous study to occur on day 2 post-wounding and infection ${ }^{34,39}$. The mouse was placed in the magnetic coil and an AMF was applied at a field amplitude and duration sufficient for in vitro biofilm disruption (i.e. $31 \mathrm{kA} / \mathrm{m}$ for $3 \mathrm{~min}$ ). Whole animal bioluminescence imaging was used to quantify $S$. aureus burden in the wound before and immediately after AMF treatment. It has been previously demonstrated that the intensity of the $S$. aureus bioluminescent signal is directly proportional to the number of bacteria quantified by an $e x$ vivo colony forming unit assay performed on mouse wounds ${ }^{35,40}$. The inoculation of MNP into the wound did not result in any change in $S$. aureus burden in the absence of AMF exposure (Fig. 5a). The extent of $S$. aureus thermal inactivation was compared between MNPs bound to specific anti-SA antibody or isotype control. Anti-SA targeting resulted in a significantly enhanced $S$. aureus inactivation (80\%) compared with non-specific IgG $(50 \%)$ or compared with MNPs with no linked antibody (p<0.05) (Fig. 5a). We next measured $S$. aureus survival in the wound bed before and for 3 days following treatment. The mice treated with AMF once at day 2 post-infection maintained a low level of wound bacterial burden, while $S$. aureus levels remained high up to day 5 post-infection in the absence of AMF treatment (Fig. 5b). As a result, wound closure in the mice with anti-SA targeted MNPs was significantly enhanced compared to either mice with IgG targeted MNPs or mice receiving no treatment (Fig. 5c). These data indicate that the enhanced $S$. aureus inactivation by anti-SA targeted MNPs correlates with enhanced wound healing. We employed noninvasive thermal imaging to measure any increase in ambient temperature of the wound. Skin temperature at the wound margin increased to a maximum of $43^{\circ} \mathrm{C}$ immediately following a 3 min AMF exposure using anti-SA targeted MNPs, and subsequently cooled to $38^{\circ} \mathrm{C}$ one minute after AMF exposure. No physical injuries or adverse events were observed for the mice following AMF treatment. A similar temperature increase within the wound was observed for anti-IgG MNPs (data not shown). 


\section{Discussion}

Recent clinical data has implicated microbial biofilms in the exacerbation of chronic wounds ${ }^{44-46}$. In this study, we developed a novel antimicrobial thermotherapy platform in which antibody-targeted MNPs were excited by a high-amplitude, high-frequency AMF. We demonstrated that this platform was highly effective at safely.

Previous studies have focused on a successful application of low-frequency RF MNP hyperthermia in cancer treatment ${ }^{14-16}$. However, to our knowledge, this is the first in-vivo application of MNP thermal therapy in which AMF excitation of antibody-bound MNPs was used to kill $S$. aureus and resolve wound infection. Due to differences in the temperature sensitivity of $S$. aureus compared to cancer cells, higher temperature transients must be safely generated for realization of this technology as an effective therapeutic for infectious pathogens. By applying a focused, high-frequency, high-amplitude magnetic field for short durations, we generated a localized increase in pathogen cell surface temperature without causing a harmful increase in ambient tissue temperature ${ }^{29}$. Using an order-of-magnitude higher frequency AMF $(\sim 2 \mathrm{MHz})$ than was previously applied for cancer therapy ( $\sim 500$ $\mathrm{kHz}$ ), we sufficiently excited iron oxide nanoparticles, and following a brief AMF field exposure (on the order of a minute), we induced sufficient heating to inactivate $S$. aureus at the interface between MNPs and $S$. aureus cells bound to them. Using short exposure times, any adverse physiological responses due to excessive exposure to a high-frequency AMF were prevented. $S$. aureus thermal inactivation occurred following a 3 minute AMF exposure using anti-SA MNPs despite a maximum measured ambient temperature of $43^{\circ} \mathrm{C}$, which is an insufficient temperature to induce $S$. aureus thermal inactivation. Recently, Dana and Gannot ${ }^{31}$ estimated that AMF-excited MNPs could generate a significant transient temperature increase within nanometers of each MNP surface, and this MNP focused heating could describe a mechanism of $S$. aureus thermal inactivation.

Using non-invasive thermal imaging, we observed very limited off-target tissue heating and no apparent tissue damage. Specifically, we observed an increase in ambient temperature from $32^{\circ} \mathrm{C}$ to $38^{\circ} \mathrm{C}$ in the tissue surrounding the wound edge, which cooled down to $35^{\circ} \mathrm{C}$ within $1 \mathrm{~min}$. The physiological mechanism of heat removal by blood circulation and convection in room air presumably contributed to the rapid cooling of the skin. A recent study by Giladi et al. ${ }^{47}$ showed that a mouse could tolerate heating from a 3 -fold higher electromagnetic field due to such processes that facilitate heat removal, while heating from the same field in vitro resulted in a significant temperature increase due to a lack of active heat removal.

A challenge for the realization of MNP thermotherapy has been administering MNPs in concentrations sufficient to facilitate adequate heating, but without causing toxicity due to oxidative stress. Previous studies determined maximum safe parenteral dosing limits ( $\sim 10$ $\mathrm{mg} / \mathrm{kg})^{24}$ based upon the assumption that nanoparticles would be continuously removed from the blood ${ }^{48,49}$. However, this dosing limit may be overcome if antibody-targeted MNPs remain bound to target pathogen cells for extended durations, providing adequate time for macrophages and the reticuloendothelial system to clear unbound MNPs ${ }^{50,51}$.

Our data revealed that anti-SA targeted MNPs exhibited greater antimicrobial activity than anti-IgG MNPs, and this enhanced effect eventually resulted in enhanced wound healing. It is noteworthy that the mice treated with anti-IgG MNPs consistently achieved $S$. aureus killing, albeit 1-fold less efficient than targeted MNP-SA. This is likely due to the heating of non-specifically accumulated MNPs that were not cleared from the wound bed. Taken together, our results suggest that antimicrobial efficiency may be enhanced while ensuring 
treatment safety by further customization of MNP/antibody combinations as well as the development of nanoparticles with even greater heat transfer properties.

There are several issues to be resolved in future studies. First, we previously reported that the tightly regulated recruitment of phagocytes (neutrophils, macrophages, etc.) to the site of infection is a critical element of innate immune protection ${ }^{34,39}$. It should be evaluated whether targeted MNP hyperthermia aids or detracts from this normal, innate immune response, and through this evaluation, it is further necessary to find suitable conditions for MNP hyperthermia that cooperate with neutrophil-mediated bacterial clearance and wound resolution. Second, bacteria have adapted a quorum sensing mechanism that plays an important role in the transition from a planktonic to biofilm stage ${ }^{52}$. It will be further important to evaluate whether this treatment modality can disrupt the quorum sensing mechanism and thereby effectively inhibit biofilm formation and growth. Third, many chronic wounds are polymicrobial with multiple bacterial species embedded in a biofilm consisting of self-secreted polysaccharide matrix ${ }^{53,54}$. This suggests that a strategy in which the polysaccharide matrix or common surface antigen is targeted may be more effective than targeting $S$. aureus alone.

Magnetic nanoparticle targeting of bacterial pathogens can be more effective than either passive immunity or other antimicrobial therapeutic modalities alone. It has been reported that multiple redundant mechanisms of immune evasion by $S$. aureus could result in recurrent infection ${ }^{6}$. The bactericidal mechanism of MNP thermotherapy is independent of this natural immunity and cell-mediated phagocytosis and may therefore be superior to antimicrobial treatments utilizing antibody or other biologics. Further, an application of our AMF can achieve sufficient tissue penetration depth ( centimeters) to target infections that are disseminated deep within tissues. Importantly, targeted thermotherapy may be successfully combined with standard-of-care antibiotic treatment for polymicrobial biofilm infections and enhance antibiotic penetration by disrupting the biofilm polysaccharide matrix. This may significantly enhance the efficacy of a given antimicrobial and facilitate reduced drug dosing, thus providing a valuable tool for the treatment of fragile patients who cannot tolerate high dose systemic antibiotics.

In summary, we have developed a magnetic antimicrobial thermotherapy platform utilizing antibody-targeted MNPs and high-frequency, high-amplitude AMFs, and we validated its efficacy in the rapid clearance of $S$. aureus pathogens in vitro and in vivo in a mouse model of skin wound infection.

\section{Acknowledgments}

We thank Dr. Thomas Graf (Center for Genomic Regulation, Barcelona Spain) and Dr. Ambros Cheung (Dartmouth Medical School) for generously providing EGFP-lys-mice and the bioluminescent strain of $S$. aureus. We also thank Dr. Jose Renau (UC Santa Cruz) for use of the FLIR SC8000 thermal camera. This research was supported by NIH AI47294 and a generous grant from the Robert S. and Star Pepper Foundation to SIS, as well as other private donors.

\section{References}

1. American Diabetes Association. Diabetes Care; Consensus Development Conference on Diabetic Foot Wound Care; 7-8 April 1999; Boston, Massachusetts. 1999. p. 1354-60.

2. Fonder MA, Lazarus GS, Cowan DA, Aronson-Cook B, Kohli AR, Mamelak AJ. Treating the chronic wound: A practical approach to the care of nonhealing wounds and wound care dressings. J Am Acad Dermatol. 2008; 58:185-206. [PubMed: 18222318]

3. Smith JA, O'Connor JJ. Nasal carriage of Staphylococcus aureus in diabetes mellitus. Lancet. 1966; 2:776-7. [PubMed: 4162327] 
4. Tuazon CU, Perez A, Kishaba T, Sheagren JN. Staphylococcus aureus among insulin-injecting diabetic patients. An increased carrier rate. JAMA. 1975; 231:1272. [PubMed: 1172956]

5. Celli J, Finlay BB. Bacterial avoidance of phagocytosis. Trends Microbiol. 2002; 10:232-7. [PubMed: 11973157]

6. Foster TJ. Immune evasion by staphylococci. Nat Rev Microbiol. 2005; 3:948-58. [PubMed: 16322743]

7. Rooijakkers SH, van Kessel KP, van Strijp JA. Staphylococcal innate immune evasion. Trends Microbiol. 2005; 13:596-601. [PubMed: 16242332]

8. Bjarnsholt T, Kirketerp-Moller K, Jensen PO, Madsen KG, Phipps R, Krogfelt K, et al. Why chronic wounds will not heal: a novel hypothesis. Wound Repair Regen. 2008; 16:2-10. [PubMed: 18211573]

9. Davies D. Understanding biofilm resistance to antibacterial agents. Nat Rev Drug Discov. 2003; 2:114-22. [PubMed: 12563302]

10. Liu C, Bayer A, Cosgrove SE, Daum RS, Fridkin SK, Gorwitz RJ, et al. Clinical practice guidelines by the infectious diseases society of america for the treatment of methicillin-resistant Staphylococcus aureus infections in adults and children. Clin Infect Dis. 2011; 52:e18-55. [PubMed: 21208910]

11. Antibiotic resistance and topical treatment. Br Med J. 1978; 2:649-50. [PubMed: 698645]

12. Monroe D. Looking for chinks in the armor of bacterial biofilms. PLoS Biol. 2007; 5:e307. [PubMed: 18001153]

13. Lee JH, Jang JT, Choi JS, Moon SH, Noh SH, Kim JW, et al. Exchange-coupled magnetic nanoparticles for efficient heat induction. Nat Nanotechnol. 2011; 6:418-22. [PubMed: 21706024]

14. Dudeck O, Bogusiewicz K, Pinkernelle J, Gaffke G, Pech M, Wieners G, et al. Local arterial infusion of superparamagnetic iron oxide particles in hepatocellular carcinoma: A feasibility and 3.0 T MRI study. Invest Radiol. 2006; 41:527-35. [PubMed: 16763472]

15. Jordan A, Scholz R, Maier-Hauff K, van Landeghem FK, Waldoefner N, Teichgraeber U, et al. The effect of thermotherapy using magnetic nanoparticles on rat malignant glioma. J Neurooncol. 2006; 78:7-14. [PubMed: 16314937]

16. Maier-Hauff K, Rothe R, Scholz R, Gneveckow U, Wust P, Thiesen B, et al. Intracranial thermotherapy using magnetic nanoparticles combined with external beam radiotherapy: results of a feasibility study on patients with glioblastoma multiforme. J Neurooncol. 2007; 81:53-60. [PubMed: 16773216]

17. Pankhurst QA, C J, Jones SK, Dobson J. Applications of magnetic nanoparticles in biomedicine. J Phys D: Appl Phys. 2003; 36:R167-R81.

18. Di Poto A, Sbarra MS, Provenza G, Visai L, Speziale P. The effect of photodynamic treatment combined with antibiotic action or host defence mechanisms on Staphylococcus aureus biofilms. Biomaterials. 2009; 30:3158-66. [PubMed: 19329182]

19. Zolfaghari PS, Packer S, Singer M, Nair SP, Bennett J, Street C, et al. In vivo killing of Staphylococcus aureus using a light-activated antimicrobial agent. BMC Microbiol. 2009; 9:27. [PubMed: 19193212]

20. Caubet R, Pedarros-Caubet F, Chu M, Freye E, de Belem Rodrigues M, Moreau JM, et al. A radio frequency electric current enhances antibiotic efficacy against bacterial biofilms. Antimicrob Agents Chemother. 2004; 48:4662-4. [PubMed: 15561841]

21. van der Borden AJ, Maathuis PG, Engels E, Rakhorst G, van der Mei HC, Busscher HJ, et al. Prevention of pin tract infection in external stainless steel fixator frames using electric current in a goat model. Biomaterials. 2007; 28:2122-6. [PubMed: 17258314]

22. Ensing GT, Roeder BL, Nelson JL, van Horn JR, van der Mei HC, Busscher HJ, et al. Effect of pulsed ultrasound in combination with gentamicin on bacterial viability in biofilms on bone cements in vivo. J Appl Microbiol. 2005; 99:443-8. [PubMed: 16108785]

23. Pitt WG, McBride MO, Lunceford JK, Roper RJ, Sagers RD. Ultrasonic enhancement of antibiotic action on gram-negative bacteria. Antimicrob Agents Chemother. 1994; 38:2577-82. [PubMed: $7872751]$ 
24. Spinowitz BS, Schwenk MH, Jacobs PM, Bolton WK, Kaplan MR, Charytan C, et al. The safety and efficacy of ferumoxytol therapy in anemic chronic kidney disease patients. Kidney Int. 2005; 68:1801-7. [PubMed: 16164657]

25. Lambrechts SA, Demidova TN, Aalders MC, Hasan T, Hamblin MR. Photodynamic therapy for Staphylococcus aureus infected burn wounds in mice. Photochem Photobiol Sci. 2005; 4:503-9. [PubMed: 15986057]

26. Ray PC, Khan SA, Singh AK, Senapati D, Fan Z. Nanomaterials for targeted detection and photothermal killing of bacteria. Chem Soc Rev. 2012; 41:3193-209. [PubMed: 22331210]

27. Cebrian G, Raso J, Condon S, Manas P. Acquisition of pulsed electric fields resistance in Staphylococcus aureus after exposure to heat and alkaline shocks. Food Control. 2012; 25:407-14.

28. Hassani M, Cebrian G, Mannas P, Condon S, Pagan R. Induced thermotolerance under nonisothermal treatments of a heat sensitive and a resistant strain of Staphylococcus aureus in media of different pH. Letters in applied microbiology. 2006; 43:619-24. [PubMed: 17083707]

29. Kennedy J, Blair IS, McDowell DA, Bolton DJ. An investigation of the thermal inactivation of Staphylococcus aureus and the potential for increased thermotolerance as a result of chilled storage. J Appl Microbiol. 2005; 99:1229-35. [PubMed: 16238754]

30. Thomas L, Dekker L, Kallumadil M, Southern P, Wilson M, Nair S, et al. Carboxylic acidstabilised iron oxide nanoparticles for use in magnetic hyperthermia. J Mater Chem. 2009; 19:6529-35.

31. Dana B, Gannot I. An analytic analysis of the diffusive-heat-flow equation for different magnetic field profiles for a single magnetic nanoparticle. Journal of Atomic, Molecular, and Optical Physics. 201210.1155/2012/135708

32. Keblinski P, Cahill DG, Bodapati A, Sullivan CR, Taton TA. Limits of localized heating by electromagnetically excited nanoparticles. Journal of Applied Physics. 2006; 100:054305.

33. Ivkov R, Bordelon DE, Cornejo C, Gruttner C, Westphal F, DeWeese TL. Magnetic nanoparticle heating efficiency reveals magneto-structural differences when characterized with wide ranging and high amplitude alternating magnetic fields. Journal of Applied Physics. 2011; 109:124904.

34. Kim MH, Liu W, Borjesson DL, Curry FR, Miller LS, Cheung AL, et al. Dynamics of neutrophil infiltration during cutaneous wound healing and infection using fluorescence imaging. J Invest Dermatol. 2008; 128:1812-20. [PubMed: 18185533]

35. Miller LS, O'Connell RM, Gutierrez MA, Pietras EM, Shahangian A, Gross CE, et al. MyD88 mediates neutrophil recruitment initiated by IL-1R but not TLR2 activation in immunity against Staphylococcus aureus. Immunity. 2006; 24:79-91. [PubMed: 16413925]

36. Amorena B, Gracia E, Monzon M, Leiva J, Oteiza C, Perez M, et al. Antibiotic susceptibility assay for Staphylococcus aureus in biofilms developed in vitro. J Antimicrob Chemother. 1999; 44:4355. [PubMed: 10459809]

37. Kennedy CA, O'Gara JP. Contribution of culture media and chemical properties of polystyrene tissue culture plates to biofilm development by Staphylococcus aureus. J Med Microbiol. 2004; 53:1171-3. [PubMed: 15496399]

38. Faust N, Varas F, Kelly LM, Heck S, Graf T. Insertion of enhanced green fluorescent protein into the lysozyme gene creates mice with green fluorescent granulocytes and macrophages. Blood. 2000; 96:719-26. [PubMed: 10887140]

39. Kim MH, Granick JL, Kwok C, Walker NJ, Borjesson DL, Curry FR, et al. Neutrophil survival and c-kit(+)-progenitor proliferation in Staphylococcus aureus-infected skin wounds promote resolution. Blood. 2011; 117:3343-52. [PubMed: 21278352]

40. Bernthal NM, Stavrakis AI, Billi F, Cho JS, Kremen TJ, Simon SI, et al. A mouse model of postarthroplasty Staphylococcus aureus joint infection to evaluate in vivo the efficacy of antimicrobial implant coatings. PLoS One. 2010; 5:e12580. [PubMed: 20830204]

41. Cho JS, Pietras EM, Garcia NC, Ramos RI, Farzam DM, Monroe HR, et al. IL-17 is essential for host defense against cutaneous Staphylococcus aureus infection in mice. J Clin Invest. 2010; 120:1762-73. [PubMed: 20364087]

42. Cho JS, Zussman J, Donegan NP, Ramos RI, Garcia NC, Uslan DZ, et al. Noninvasive in vivo imaging to evaluate immune responses and antimicrobial therapy against Staphylococcus aureus and USA300 MRSA skin infections. J Invest Dermatol. 2011; 131:907-15. [PubMed: 21191403] 
43. Daum RS, Spellberg B. Progress toward a Staphylococcus aureus vaccine. Clin Infect Dis. 2012; 54:560-7. [PubMed: 22186773]

44. Donlan RM, Costerton JW. Biofilms: survival mechanisms of clinically relevant microorganisms. Clin Microbiol Rev. 2002; 15:167-93. [PubMed: 11932229]

45. Parsek MR, Singh PK. Bacterial biofilms: an emerging link to disease pathogenesis. Annu Rev Microbiol. 2003; 57:677-701. [PubMed: 14527295]

46. Dowd SE, Wolcott RD, Sun Y, McKeehan T, Smith E, Rhoads D. Polymicrobial nature of chronic diabetic foot ulcer biofilm infections determined using bacterial tag encoded FLX amplicon pyrosequencing (bTEFAP). PLoS One. 2008; 3:e3326. [PubMed: 18833331]

47. Giladi M, Porat Y, Blatt A, Shmueli E, Wasserman Y, Kirson ED, et al. Microbial growth inhibition by alternating electric fields in mice with Pseudomonas aeruginosa lung infection. Antimicrob Agents Chemother. 2010; 54:3212-8. [PubMed: 20547811]

48. Bucci OM. Electromagnetism, nanotechnologies and biology: New challenges and opportunities. Electromagnetic Theory (EMTS) on 2010 URSI International Symposium. 2010:77-80.

49. Ivkov R, DeNardo SJ, Daum W, Foreman AR, Goldstein RC, Nemkov VS, et al. Application of high amplitude alternating magnetic fields for heat induction of nanoparticles localized in cancer. Clin Cancer Res. 2005; 11:7093s-103s. [PubMed: 16203808]

50. Luciani N, Wilhelm C, Gazeau F. The role of cell-released microvesicles in the intercellular transfer of magnetic nanoparticles in the monocyte/macrophage system. Biomaterials. 2010; 31:7061-9. [PubMed: 20619790]

51. Jain TK, Reddy MK, Morales MA, Leslie-Pelecky DL, Labhasetwar V. Biodistribution, clearance, and biocompatibility of iron oxide magnetic nanoparticles in rats. Mol Pharm. 2008; 5:316-27. [PubMed: 18217714]

52. Wintermute EH, Silver PA. Dynamics in the mixed microbial concourse. Genes Dev. 2010; 24:2603-14. [PubMed: 21123647]

53. Han A, Zenilman JM, Melendez JH, Shirtliff ME, Agostinho A, James G, et al. The importance of a multifaceted approach to characterizing the microbial flora of chronic wounds. Wound Repair Regen. 2011; 19:532-41. [PubMed: 22092791]

54. Edwards R, Harding KG. Bacteria and wound healing. Curr Opin Infect Dis. 2004; 17:91-6. [PubMed: 15021046] 


\section{Conjugation of MNP with antibody}

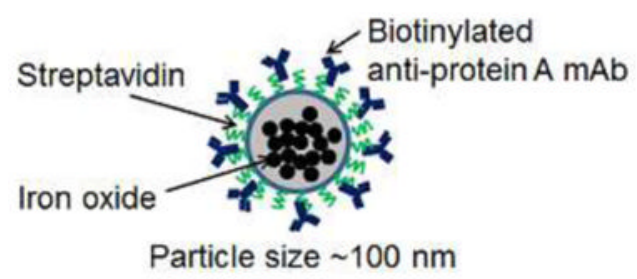

4. Targeted thermal disruption of bacterial wall by localized MNP heating on AMF application

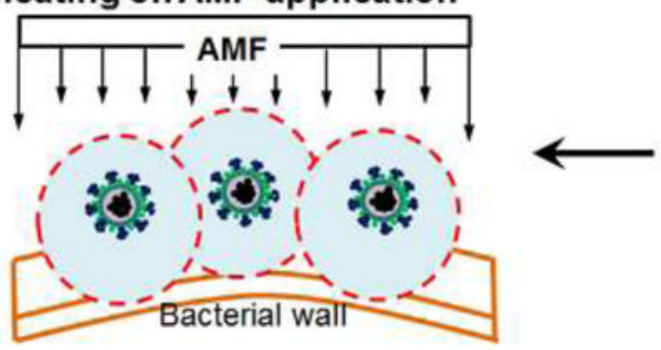

2. Local injection of MNP-conjugates into the infected wound

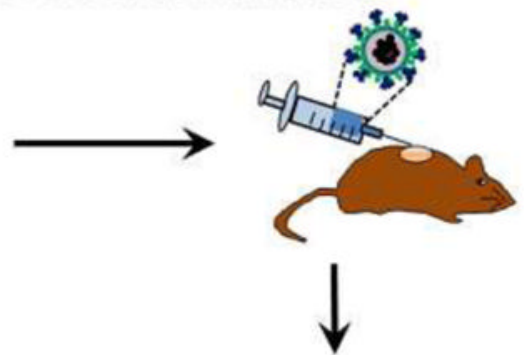

3. Targeted specific binding of MNP-conjugates to bacterial wall

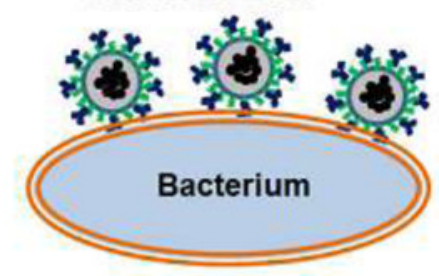

Figure 1.

Schematic of thermotherapy platform utilizing antibody-targeted, magnetic nanoparticles. 
a
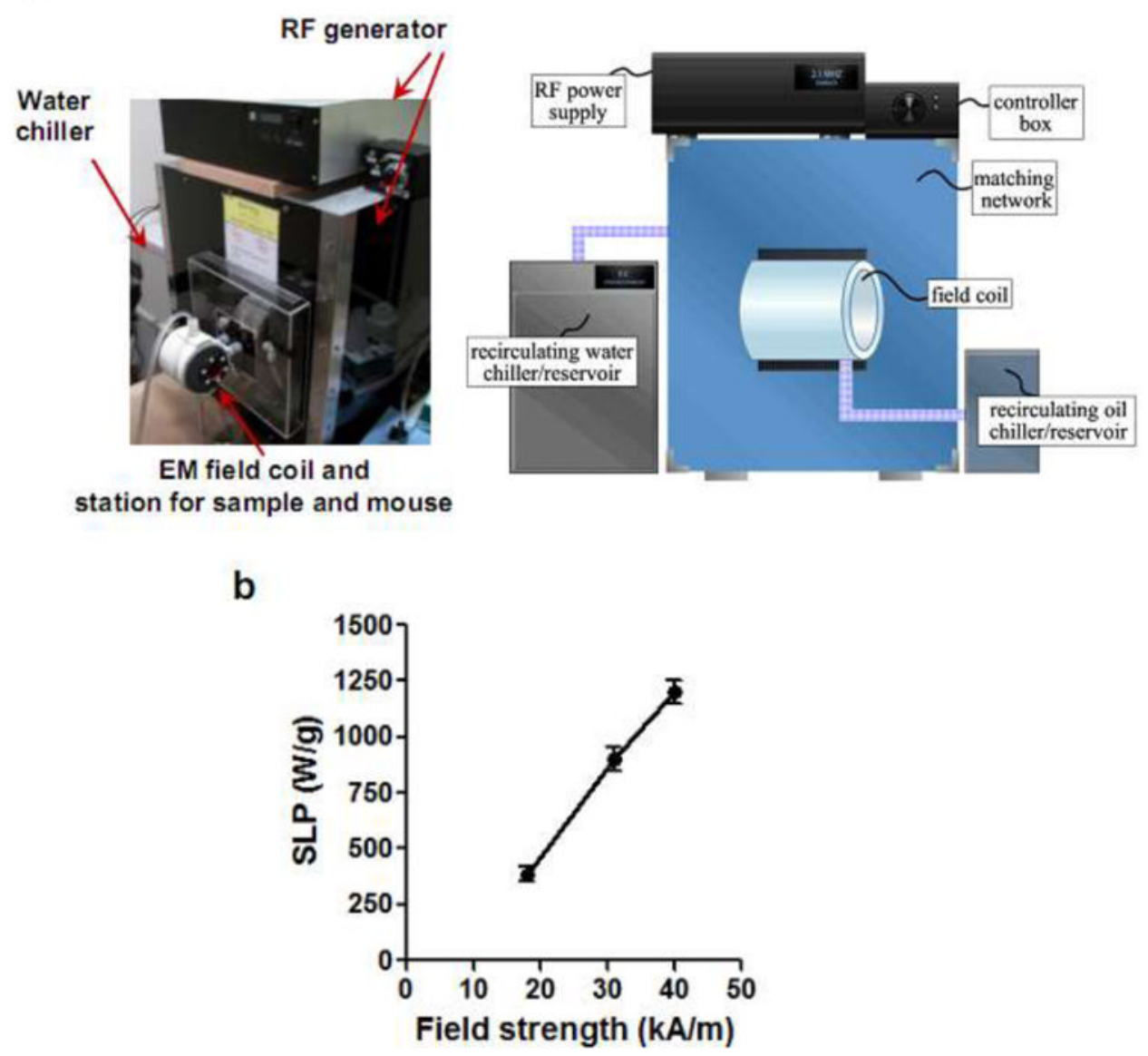

Figure 2. High-frequency, high field amplitude electro-magnetic field generator for antimicrobial thermotherapy platform

(a) Photographic image and schematic of custom built high-frequency generator platform incorporating electromagnetic coils (40 mm inner diameter). (b) Experimentally measured SLP values of $100 \mathrm{~nm}$ iron oxide nanoparticles at different magnetic field amplitudes $(\mathrm{n}=3)$. 
a

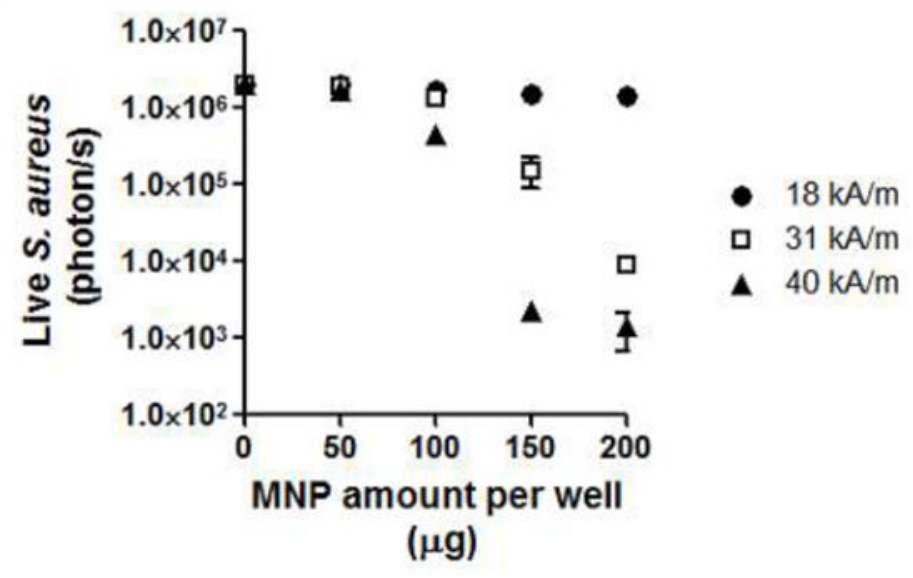

b

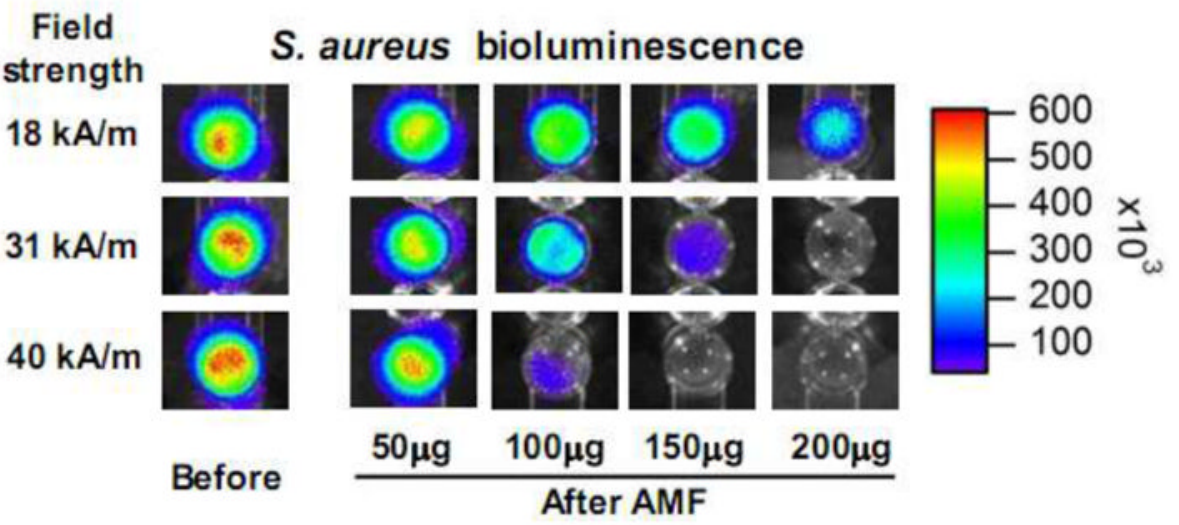

Figure 3. Efficacy of high amplitude AMF on in vitro $S$. aureus biofilm ablation

(a) $S$. aureus killing curve following AMF application ( $3 \mathrm{~min}$ ) as a function of MNP dose (0-200 $\mu \mathrm{g}$ per well) and AMF amplitude $(18,31$, and $40 \mathrm{kA} / \mathrm{m})$. (b) Representative bioluminescent image of $S$. aureus biofilm on 96 well plate before and after AMF application. 
a

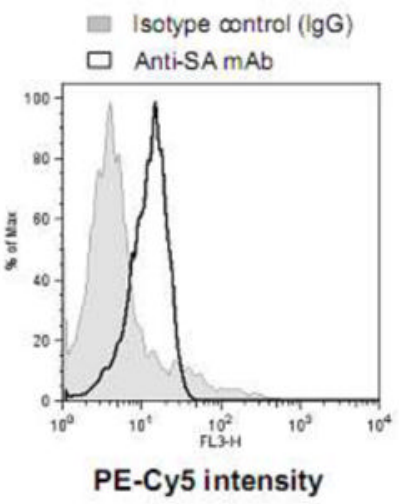

C

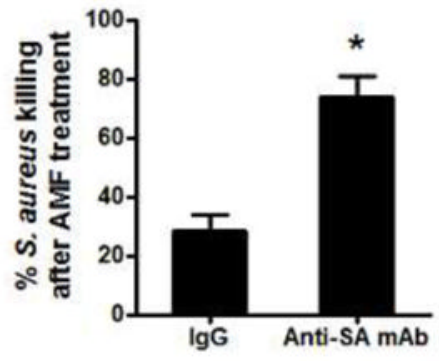

b

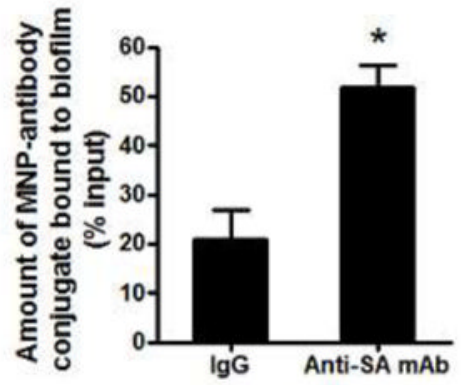

d

Green/ SYTO9=live cells Red/PI=Dead cells

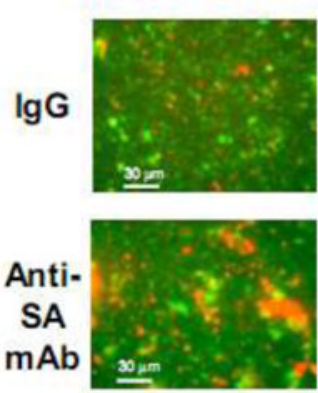

Figure 4. Antibody conjugation of MNP, specific targeting, and in vitro disruption of $S$. aureus biofilm

(a) Representative flow cytometric histogram demonstrating specific binding of anti-protein A monoclonal antibody (anti-SA $\mathrm{mAb}$ ) to $S$. aureus. $S$. aureus bacteria were incubated with PE-Cy5 conjugated anti-SA mAb or isotype control and events were analyzed for PE-Cy5 fluorescence intensity (n=3). (b) The binding efficiency of MNP-antibody conjugate to $S$. aureus biofilm. A defined amount of MNP-anti-SA mAb $(200 \mu \mathrm{g})$ or MNP-IgG conjugate $(200 \mu \mathrm{g})$ was added to the biofilm cultured on 96 well plates and incubated for $2 \mathrm{hrs}$. The binding efficiency was determined by normalizing the quantity of MNPs remaining in the well after washing out unbound MNPs $(\mathrm{n}=6)$. (c) Comparison of the extent of $S$. aureus killing for MNPs conjugated with anti-SA versus MNPs conjugated with a non-specific isotype control following AMF treatment $(31 \mathrm{kA} / \mathrm{m}$ for $3 \mathrm{~min})$ using in vitro biofilm culture assay in 96 well plate $(\mathrm{n}=5)$. The extent of $S$. aureus biofilm killing was determined based on quenching of bioluminescence signal before and after AMF treatment. (d) Representative SYTO9/PI fluorescence images and quantification of $S$. aureus viability following AMF treatment for MNPs conjugated with anti-SA versus MNPs conjugated with a non-specific isotype control (green/SYTO9 = live cells; red/PI = dead cells $)(n=3)$. *, p<0.05 vs IgG control group. 
a

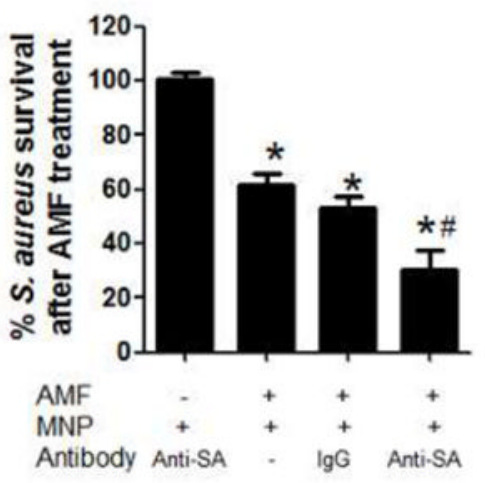

S. aureus bioluminescence (photon/sec)

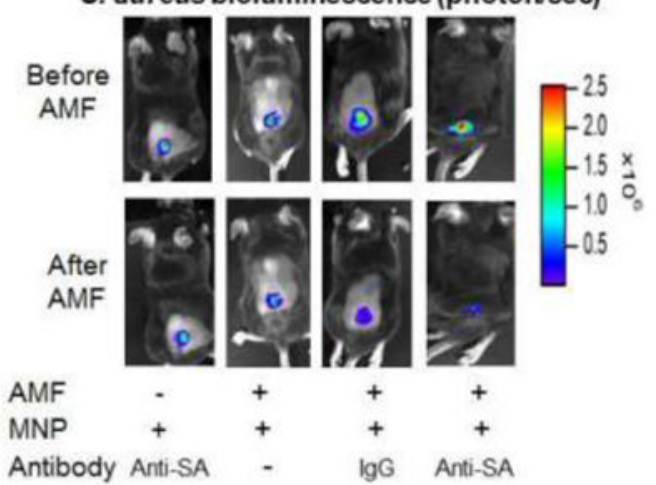

b

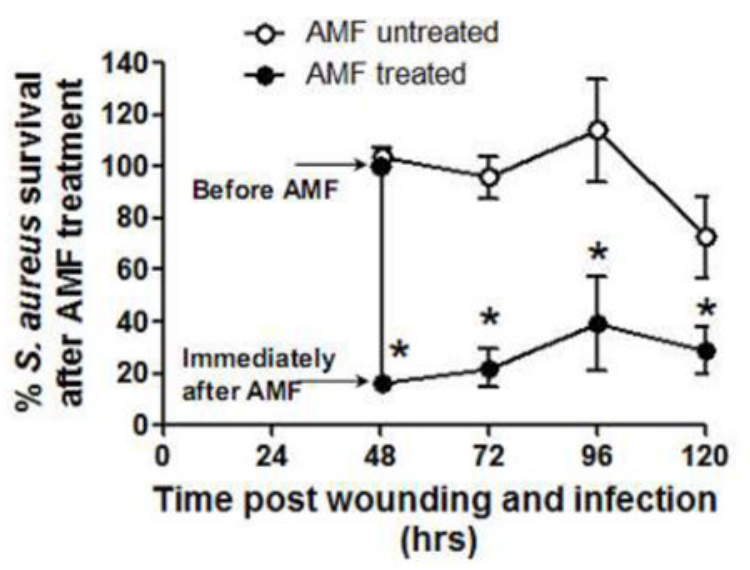

Figure 5. In vivo validation of antibody-targeted thermal inactivation of $S$. aureus infection in a mouse skin wound

Mice were infected with $S$. aureus $\left(1 \times 10^{7} \mathrm{CFU}\right)$ at wound sites and $200 \mu \mathrm{g}$ of MNPantibody conjugates were locally injected into wound at day 2 post-infection. Then, an AMF was applied for $3 \mathrm{~min}(31 \mathrm{kA} / \mathrm{m}$ field amplitude, $2.1 \mathrm{MHz}$ frequency). Mice were divided into four experimental groups: (1) mice injected with MNP-anti-SA mAb conjugate but without AMF treatment, (2) mice injected with MNP only and with AMF treatment, (3) mice injected with MNP-IgG conjugate and with AMF treatment, (4) mice injected with MNP-anti-SA mAb conjugate and with AMF treatment. (a) Representative bioluminescence images of mice and quantification before and immediately after AMF treatment $(n=6)$. (b) Comparison of a time course of $S$. aureus burden in the wound between $S$. aureus $\left(1 \times 10^{7}\right.$ CFU) infected mice treated with or without AMF exposure. $200 \mu \mathrm{g}$ of MNP-anti SA mAb conjugates were injected for both groups of mice. The AMF was applied once at day 2 postinfection, and $S$. aureus burden was determined by bioluminescence imaging daily. (c)

Representative photographic images of wound area and normalized wound size before and at 3 day post-AMF treatment $(n=5)$. Wound size on day 3 post-AMF treatment was normalized with respect to the baseline value before AMF treatment on day 2 post-infection. Dotted line indicates boundary of wound edge. (d) Representative infrared thermal imaging of mouse treated with MNP-anti-SA mAb conjugate before and after AMF treatment and quantification. Dotted line indicates boundary of wound edge. Ambient temperature measured by thermal imaging within wound area and surrounding non-wound area $(n=3)$ * , 
$\mathrm{p}<0.05$ vs mice injected with MNP-antibody conjugate but without AMF treatment. \#, $\mathrm{p}<0.05$ vs mice injected with MNP-IgG conjugate and with AMF treatment. 